\begin{abstract}
Akiko Mogi ${ }^{1 \star}$, Yasuyuki Takahashi ${ }^{2}$, Kimiko Nakajima ${ }^{3}$, Hiroshi Shimizu ${ }^{1}$, Kyoko Saito $^{2}$ and Ken-ichi Tomaru ${ }^{1}$

${ }^{1}$ Department of Ragiological Technology, Gunma Children's Medical Hospital, 779 Shimohakoda, Hokkitsu-machi, Shibukawa 377-8577, Japan ${ }^{2}$ Department of Nuclear Medicine Technology, Gunma Prefectural College of Health Sciences, Maebashi, Japan

${ }^{3}$ Department of Pediatrics, Gunma Children's Medical Hospital, Shibukawa, Japan
\end{abstract}

Dates: Received: August 20, 2014; Accepted: September 13, 2014; Published: September 11, 2014

*Corresponding author: Akiko Mogi, Department of Ragiological Technology, Gunma Children's

Medical Hospital, 779 Shimohakoda, Hokkitsu-machi, Shibukawa 377-8577, Japan, Tel: 81279523511; Fax: +81279522045; E-mail: loo3na@gmail.com

www.peertechz.com

Keywords: Pediatric myocardial perfusion SPECT Correction method; EANM paediatric dosage card

ISSN: 2455-2976

\section{Research Article \\ Effect of Scatter, Attenuation and Resolution Correction on a Pediatric Myocardial Perfusion SPECT Image}

\section{Introduction}

The image processing of pediatric nuclear medicine images has not been widely discussed. The patient size can differ considerably. And the target organ is usually small. Also, the SPECT acquisition time may be limited to the time the patient can be kept asleep.

A ${ }^{99 \mathrm{~m}} \mathrm{Tc}$ radiopharmaceutical dose for pediatric myocardial SPECT has recently been recommended by the European Association of Nuclear Medicine (EANM) [1], and it is lower than the one that has conventionally been used up until now. On the dosage card of the EANM, the radiopharmaceutical dose differs by the weight of the child. And image acquisition condition was reconsidered recently, too [2]. However, the pediatric patients are not considered at all.

The differences for imaging a child compared to imaging an adult are: 1) a smaller radius of rotation, 2) less attenuation, and 3) possibly less scatter. However, the effect of these differences on standard corrections has not been investigated for the pediatric case until now. In this study, image processing is chosen and used by a scatter correction, an attenuation correction, and a resolution correction to achieve a low-noise image for a child. The independent variable in the study will be the projection count density.

\section{Materials and Methods}

The SPECT system used was the PRISM-AXIS (SHIMADZU Co., Kyoto, Japan) and E-CAM (Toshiba Medical Systems, Tochigi, Japan). We employed the continuous-mode at $6^{\circ}$ intervals over $360^{\circ}$ (10counts/pixel/projection, 10rotations in total). The matrix size was $64 \times 64$, and the image reconstruction pixel size was $3.2 \times 3.2 \mathrm{~mm}$. The data processor was the Odyssey-FX (SHIMADZU Co., Kyoto, Japan) and GMS-5500A/PI (Toshiba Medical Systems, Tochigi, Japan).

The phantom studies achieved 10counts/pixel/projection/rotation for the target area. Continuous mode acquisition was employed. Data from multiple sequential rotations were added together to provide data sets with from 10 to 100 counts/pixel/projection. Note that 100 counts/pixel/projection is the standard adult count density.

A three year-old boy presented with a history of hypo plastic left heart syndrome (HLHS).Echocardiogram performed on depressed right ventricular systolic function. ECG confirmed complete right 
bundle branch block and right at rial overload. The boy had two times operation, the first was pulmonary artery banding, Norwood procedure, right ventricle- pulmonary artery conduit, and the second was Bidirectional Glenn procedure, pulmonary artery plasty. The boy was height of $56 \mathrm{~cm}$, weight of $12.0 \mathrm{~kg}$, and chest circumference of $39 \mathrm{~cm}$. The myocardial perfusion SPECT of $90 \mathrm{~min}$ after intravenous injection of $87.9 \mathrm{MBq}$ of ${ }^{99 \mathrm{~m}} \mathrm{Tc}$-tetrofosmin at rest was also examined. SPECT scanning was approved ethically. The boy did not reach 18 years; it was started after the contents from parents. The anesthesia technique was not used.

Reconstruction was based on an implementation of ordered subsets-EM (OS-EM) algorithm [3,4]. A Butterworth filter (order 7, cutoff frequency $=0.37 \mathrm{cycles} / \mathrm{cm}$ ) was used as a pre-filter.

\section{Scatter correction}

We chose the TEW scatter correction method [5]. The effect of the scatter correction was tested on a cold-rod phantom. The shell of the phantom was $13.0 \mathrm{~cm}$ in height and $18.0 \mathrm{~cm}$ in diameter. Within the phantom, $16,14,12,10$, and 8 , and $6 \mathrm{~mm}$ diameter cold rods were placed in different sectors that were located adjacent to one another (Figure 1, upper right). We compared the correction effect for each level of count density.

\section{Attenuation correction}

We chose the method called the scatter and photo peak window data for our Attenuation Correction (SSPAC) technique [6] rather than employ a correction based on an X-ray CT. This choice was made to avoid increasing the radiation exposure to the pediatric patient. Here we are assuming that such an increase is probably even more important for a young patient than it is for an adult patient. The phantom was an elliptical cylindrical phantom that had an 18.0 $\mathrm{cm}$ long axis and a $13.0 \mathrm{~cm}$ short axis (Figure 2, upper right). It was uniformly filled with ${ }^{99 \mathrm{~m}} \mathrm{Tc}$.

For SSPAC images [6], a triple peak window scatter correction was performed by the scatter data obtained from the lower side of the main photo peak window. This method is incorporated each

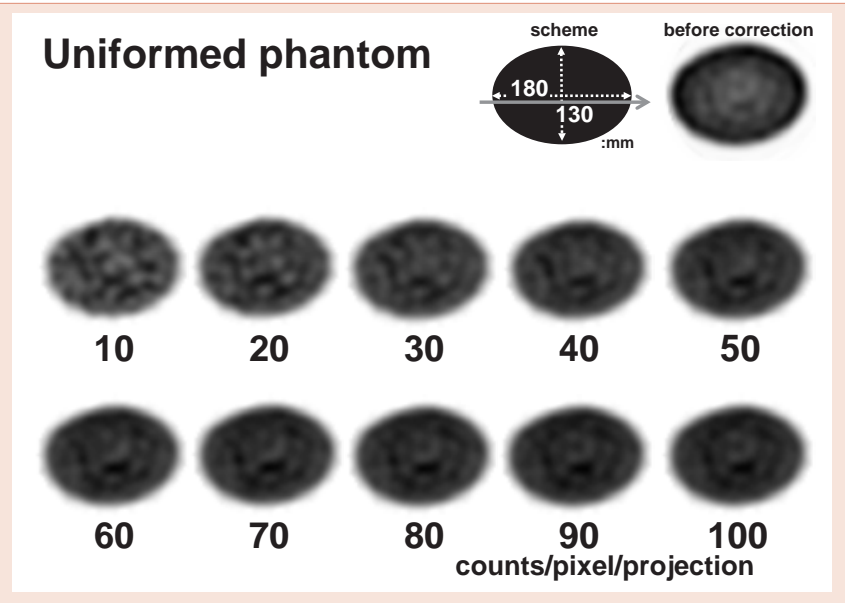

Figure 1: SPECT image with before and after TEW scatter correction. SPECT images for the evaluation of a uniform phantom with a change in the counts per pixel per projection (from 10to 100 counts / pixel / projection).

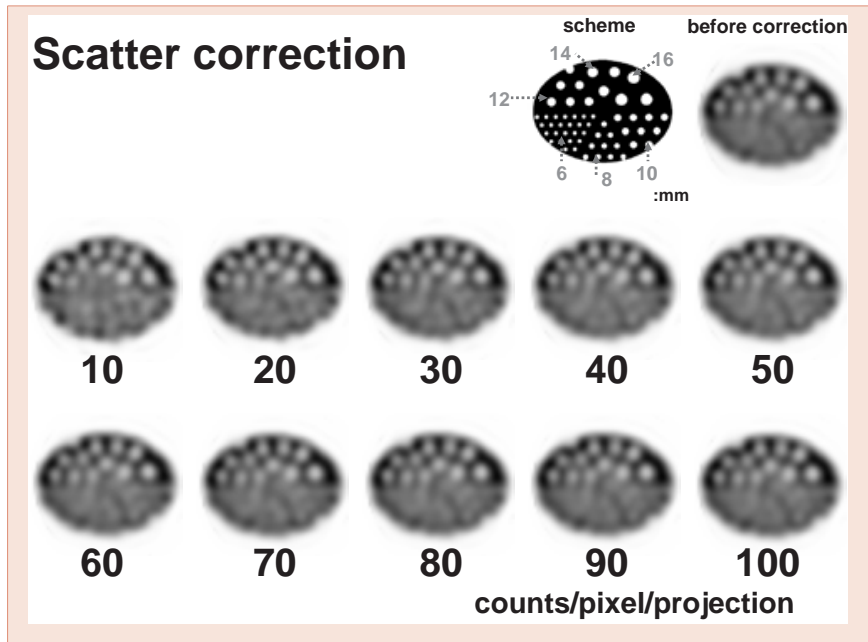

Figure 2: SPECT images before and after SSPAC scatter correction. Images are of the cold-rod phantom with a change in the counts per pixel per projection (from 10to 100 counts / pixel / projection).

attenuation coefficient that the segment method which sorts out the body outline, the lungs, the mediastinum from Compton scattered image. The attenuation correction images were reconstructed by Chang's iterative method [7] using the attenuation coefficient map. This phantom used a correction coefficient of the mediastinal part.

\section{Resolution correction}

We chose the resolution correction method called the Collimator Broad Correction (CBC) [8]. We constructed an original pediatric myocardium phantom that had the shape of a frustum; a base diameter of $4 \mathrm{~cm}$ and a height of $4.5 \mathrm{~cm}$ were employed. The myocardium thickness was $0.9 \mathrm{~mm}$ (Figure 3 , upper right). This size of the phantom set the left ventricular end- diastolic volume (LVEDV) and LV wall thickness from the BSAvalues (Dubos-type, Data disseminationis V-link co., Ltd, Tokyo, Japan) for the average weight and average height for a child. This age group has much postoperative follow-up. The phantom was imaged with a 15 -mm-diameter circular defect set into the anterior wall. The activity of the background was $1 / 10$ of that in the myocardium.

\section{Statistics}

The individual SPECT images are displayed after normalizing the maximum count of each ECT image to $100 \%$.

In the experiments using elliptical cylinders and the pediatric myocardial phantom, the normalized mean square error (NMSE, $\left[\Sigma(\mathrm{Xi}-\mathrm{Oi})^{2} / \Sigma \mathrm{Oi}^{2}\right]$; Xi, measurement image $(10 \sim 90$ counts/ pixel/projection); Oi, standard image (100 counts/pixel/projection); i pixel number $(\mathrm{i}=1-\mathrm{n})$ ) was determined.

\section{Results}

For the scatter correction (Figure 1), attenuation correction (Figure 2), and resolution correction (Figure 3) there is the expected result that the image becomes less "noisy" as the count density increases.

For $10 \sim 90$, and 100 counts/pixel/projection, the stability of the 
phantom image compared it in NMSE (Figure 4). And the uniformed in the SPECT image (Figure 1) was expressed by a normalized mean value \pm standard deviation (\%; grey arrow of the scheme). In each 10 , $20,30,40,50,60,70,80,90$ and 100 counts/pixel/projection data was $80.3 \pm 7.4,88.9 \pm 5.0,89.2 \pm 4.5,91.1 \pm 4.4,91.5 \pm 4.0,92.3 \pm 3.9,92.9$ $\pm 3.8,93.0 \pm 3.6,93.4 \pm 3.5$, and $94.3 \pm 3.4$.

Figure 5 shows the results for the3-year-old patient with SPECT images. In 10 counts/pixel/projection images, body (lung) artifacts and myocardial distortion were observed on the transverse image, and only myocardial (posterior) distortion was observed in 50 counts/ pixel/projection images. In 70 counts/pixel/projection images, no artifact was observed on the transverse image. By the phantom study, the SPECT was finished for shortening at acquisition time.

\section{Discussion}

The system spatial resolution of the Anger-type gamma camera is approximately $10 \mathrm{~mm}$. However, the detectability of this system is reduced in pediatric nuclear cardiology. Enlarged image scanning is possible; however, it results in a decreased image count and a longer acquisition time. The image processing techniques is not reported about pediatric SPECT reconstructions until now. This study aims to suggest practical image processing techniques to improve pediatric SPECT reconstructions. We reviewed the appropriate means to use for scatter, attenuation, and resolution correction in a pediatric patient.

First, for scatter correction, we considered the dual energy window subtraction (DEWS) method [9], the effective scatter source estimation (ESSE) method [10], and the TEW method [6]. When the source activity is not distributed uniformly, accuracy decreases with the DEW method. The ESSE method is not that suitable for pediatric patients because they can vary considerably in body size. For this reason, we chose the TEW method. This method actual surveys the scattered radiation that occurrsfor the size and shape of a particular child's body.

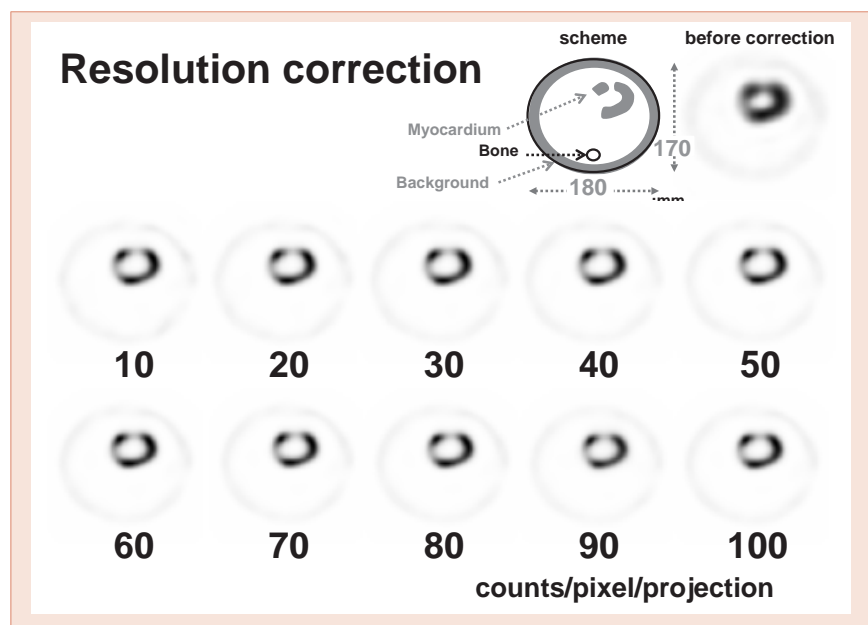

Figure 3: SPECT images before and after $\mathrm{CBC}$ attenuation correction. Images are of the pediatric myocardium with a change in the counts per pixel per projection (from 10to 100 counts / pixel / projection).

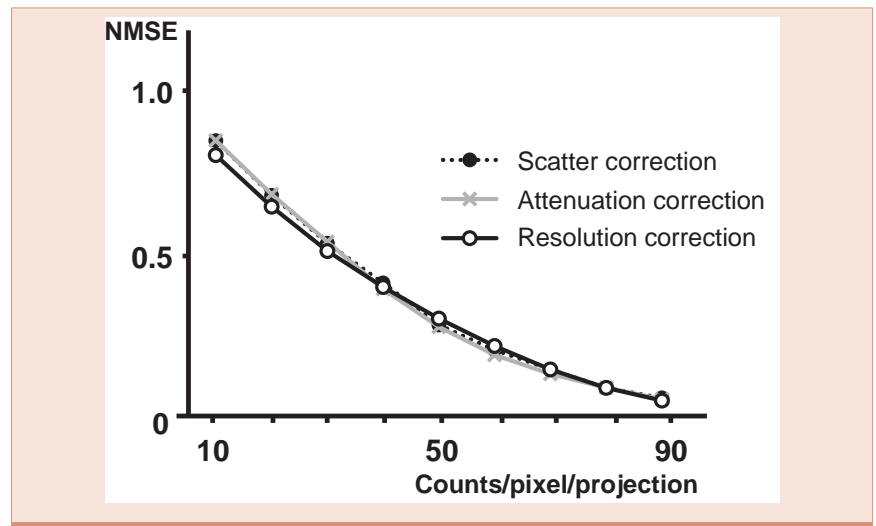

Figure 4: The NMSE metric for images with various count per pixel per projection (from 10to 100 counts / pixel / projection). The metric is calculated before and after correction separately using the image obtained from the uniformity, cold-rod and pediatric myocardial phantom.

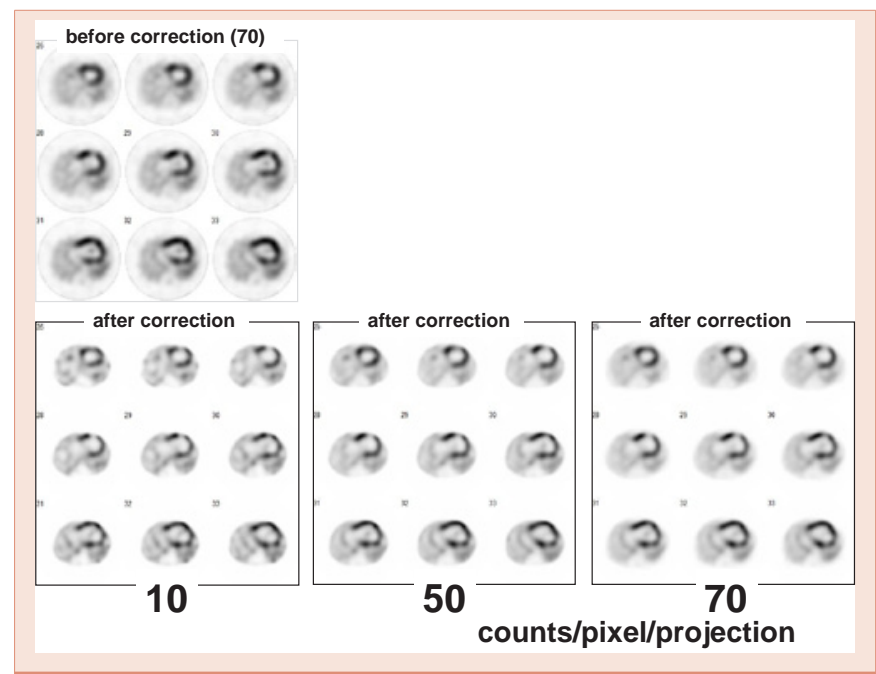

Figure 5: ${ }^{99 m} T c$-tetrofosmin myocardial SPECT images showing pediatric study. Different adjacent slicesare shown before (upper) and after (lower) all three corrections. The counts of lower three imagesare 10, 50, and 70counts/ pixel/projection.

The second is attenuation correction. Chang method [11], SSPAC method, and X-ray CT method [12] is spread. But, the additional radiation exposure by the X-rays from the CT are especially undesirable for the pediatric patient. Moreover, there are many gamma cameras in use that are not equipped with attached X-ray CT equipment. Other hands, the Chang method is useful for the uniform attenuation of the head.

The third is resolution correction. Frequency-Distance Relationship (FDR) method [13] and CBC [8] method is spread. We did not choose the FDR method because it was only two-dimensional and was not commercially available.

\section{Conclusion}

We have seen that image quality deteriorates for a rapid, lowcount acquisition, as a compromise between image quality and practicality, we recommend at least 70 counts/pixel/projection to obtain the desirable low-noise image. 


\section{Acknowledgements}

This work was supported in part by the Gunma Prefectural College of Health Sciences and Regional Coordination Center.

\section{References}

1. Lassmann M, Biassoni L, Monsieurs M, Franzius C, Jacobs F (2007) The new EANM paediatric dosage card. Eur JNucl Med Mol Imag 34: 796-798.

2. Hesse B, Tagil K, Cuocolo A, Anagnostopoulos C, Bardies M, et al. (2005) EANM/ESC procedural guidelines for myocardial perfusion imaging in nuclear cardiology. Eur J Nucl Med Mol Imaging 32: 855-897.

3. Takahashi Y, Murase K, Mochizuki T, Higashino H, Sugawara Y, et al. (2003) Evaluation of the number of SPECT projections in the ordered subsetsexpectation maximization image reconstruction method. Ann Nucl Med 17: 525-530.

4. Hudson HM, Larkin RS (1994) Accelerated image reconstruction using ordered subsets of projection data. IEEE Trans Med Imaging MI 13: 601-609.

5. Ogawa K (1994) Simulation study of triple-energy-window scatter correction in combined TI-201, Tc-99m SPECT. Ann Nucl Med 8: 277-281.

6. Yamauchi Y, Kanzaki Y, Otsuka K, Hayashi M, Okada M, et al. (2014) Novel attenuation correction of SPECT images using scatter photo peak window data for the detection of coronary disease. J Nucl Cardiol 21: 109-117.
7. Hashimoto J, Ogawa K, Kubo A, Ichihara T, Motomura N, et al. (1998) Apprication of transmission scan-based attenuation compensation to scattercorrected thallium-201 myocardial single-photon emission tomographic images. Eur J Nucl Med 25: 120-127.

8. Takahashi $\mathrm{Y}$, Murase $\mathrm{K}$, Mochizuki $\mathrm{T}$, Sugawara $\mathrm{Y}$, Maeda $\mathrm{H}$, et al. (2007) Simultaneous three-dimensional resolution correction in SPECT reconstruction using OS-EM algorithm. J Nucl Med Tech 35: 34-38.

9. Jaszczak RJ, Greer KL, Floyd CE Jr, Harris CC, Coleman RE (1984) Improved SPECTquantification using compensation for scattered photons. J Nucl Med 25: 893-900.

10. Frey EC, Tsui BMW (1996) A new method for modeling the spatially-variant, object-dependent scatter response, function in SPECT. IEEE Nucl Sci Symp 2: 1082-1086.

11. (1978) L-T Chang, "A method for attenuation correction in radionuclide computed tmography,"IEEE Transactions on nuclear Science 25: 638-643.

12. Patton JA, Delbeke D, Sandler MP (2000) Image fusion using an integrated dual-Head coincidence camera with X-ray tube-based attenuation maps. J Nucl Med 41: 1364-1368.

13. Edholm PR, Lewitt RM, Lindholm B (1986) Novel properties of the Fourier decomposition of the sonogram. Proc SPIE 671: 8-18. 